\title{
On Classifications of Normal and Osculating Curves in 3-dimensional Sasakian Space
}

\author{
Mihriban Alyamaç Külahc1*, Mehmet Bektaş and Ahmet Bilici
}

\begin{abstract}
This study provides the definition of normal and osculating curves in 3-dimensional Sasakian space with their characterizations. Furthermore, the differential equations obtained from these characterizations are solved and their figures are presented in the text.
\end{abstract}

Keywords: Normal curve, osculating curve, differential equation.

AMS Subject Classification (2010): 53C25; 53A04.

${ }^{*}$ Corresponding author

\section{Introduction}

Sasakian manifolds were introduced in 1960 by the Japenese geometer Shigeo Sasaki [19]. There was not much activity in this field after the mid-1970s, until the advent of String theory. Since then Sasakian manifolds have gained importance in physics and geometry. For physicists and geometers, the study of Sasakian space has its own interest, so it has been extensively studied area of scientific researchs $[1,3,4,7,9,20]$.

In the 3-dimensional Sasakian space, to each regular curve $\gamma$, it is possible to associate three mutually ortogonal unit vector fields. The vectors $V_{1}, V_{2}, V_{3}$ are called the tangent, the principal normal and the binormal vector field, respectively. The planes spanned by the vector fields, $\left\{V_{1}, V_{2}\right\},\left\{V_{1}, V_{3}\right\}$ and $\left\{V_{2}, V_{3}\right\}$ are defined as the osculating plane, the rectifying plane and the normal plane, respectively. In the Euclidean space $E^{3}$, the notion of rectifying curves was introduced by Chen in [10]. In addition, he showed in [11] that there exist a simple relationship between the rectifying curves and centrodes, which play some important roles in mechanics, kinematics as well as differential geometry.

On the other hand, it should be noted that most papers on rectifying, normal and osculating curves. Because it is a quite interesting problem to obtain explicit parameter equations of rectifying, normal and osculating curves in different spaces.

In [2], Ali and Önder defined a spacelike rectifying curve with spacelike principal normal in the Minkowski spacetime $E_{1}^{4}$ and characterized such curves in terms of their curvature. Güngör and Tosun investigated spatial quaternionic rectifying curves in Euclidean space $R^{3}$ and $R^{4}$ in [13]. Also, in [16], İlarslan and Nesovic defined the spacelike, the timelike and the null rectifying curves in the Minkowski 3-space in terms of centrodes. Furthermore, İlarslan, Nesovic and Petrovic-Torgasev studied non-null and null rectifying curves, lying fully in the Minkowski 3-space $E_{1}^{3}$ and gave some parametrizations of rectifying curves in [17].

In addition to rectifying curve, normal and osculating curves are also studied by many mathematicians. In [8], Camc1, Kula and İlarslan characterized surface curves in terms of geodesic curvature, normal curvature and geodesic torsion. Also they gave some characterization for the regular surface by using the concept of transversality of surfaces in Euclidean 3-space. Moreover, Grbovic and Nesovic obtained explicit parameter equations of spacelike rectifying curves in $E_{1}^{3}$ whose projection onto spacelike, timelike and lightlike plane of $E_{1}^{3}$ is a normal curve and they also obtained explicit parameter equations of spacelike normal curves in $E_{1}^{3}$ whose projection onto lightlike plane of $E_{1}^{3}$, with respect to a chosen screen distribution, is a rectifying W-curve in [12]. In [14], İlarslan gave some

Received : 01-02-2019, Accepted : 12-04-2019 
characterizations of spacelike normal curves with spacelike, timelike or null principal normal in the Minkowski 3space $E_{1}^{3}$. Besides, İlarslan and Nesovic characterized timelike and null curves for which the position vector always lies in their normal plane in the Minkowski 3-space in [15]. In [18], Öztekin and Öğrenmiş studied normal and rectifying curves in pseudo-Galilean space $G_{1}^{3}$. In addition, Yılmaz and Külahcı gave classifications of quaternionic osculating curves in [21].

To the best of authors' knowledge, normal and osculating curves are not defined in 3-dimensional Sasakian space. The main goal of this paper is to obtain some results of these curves in Sasakian space.

The remainder of this paper is organized as follows: In section 2, the basic notion and properties are reviewed. In section 3, by considering a casual character of a curve, we gave some characterizations of normal and osculating curves in the 3-dimensional Sasakian space. We solved the differential equations of normal and osculating curves and we draw their figures as shown in Figures 1 and 2.

\section{Preliminaries}

In this paper, the notations and concepts are used from the literature $[5,6,7]$.

If a (2n+1)-dimensional differentiable manifold $\mathrm{M}$ carries a global differential 1-form $\eta$ such that

$$
\eta \wedge(d \eta)^{n} \neq 0
$$

everywhere on $M$, it is called a contact manifold and $\eta$ is a contact structure [5]. It is well known that there exists a unique vector field $\xi$ such that $\eta(\xi)=1$ and $d \eta(X, \xi)=0$. Unique vector field $\xi$ is called a characteristic vector field of $\eta$. D is said to be a contact distribution [3]

$$
D=\{X \in \chi(M): \eta(X)=0\} .
$$

It is known that the maximum dimension of integral sub-manifold is only $\eta$ for a contact manifold. A 1-dimensional integral sub-manifold is called a Legendre curve, which is necessary for avoiding the confusion with an integral curve of the vector field $\xi$. When $\phi, \eta$ and $\xi$ are of type $(1,1),(0,1)$ and $(1,0)$, respectively, satisfying that

$$
\phi^{2} X=-X+\eta(X) \xi
$$

where $\eta(\xi)=1$, then $(\phi, \eta, \xi)$ is called an almost contact manifold on $M$. Belkhelfa et al. [4] have defined an almost contact manifold, contact manifold and Sasakian manifold in Lorentzian manifold. Let $M$ be a $(2 n+1)$-dimensional differentiable manifold then in this case, $(M, \phi, \xi, \eta, g, \varepsilon)$ is called an almost contact metric manifold if $(\phi, \xi, \eta)$ is an almost contact structure on $M$ and there exists a metric $g$ such that

$$
g(\phi X, \phi Y)=g(X, Y)-\varepsilon \eta(X) \eta(Y)
$$

where $g(\xi, \xi)=\varepsilon= \pm 1$. When $\varepsilon=1$ and $\varepsilon=-1$, then $g$ is a Riemannian and Lorentzian metric, respectively. So one can say that $(\phi, \xi, \eta, g, \varepsilon)$ is a contact metric structure on $\mathrm{M}$ if it is an almost contact metric structure on $\mathrm{M}$ under the condition $d \eta(X, Y)=\varepsilon g(X, \phi Y)$ [4]. It is well known that every contact metric manifold is a contact manifold [5].

Let $M$ be a $(2 n+1)$-dimensional almost contact manifold with almost contact structure $(\phi, \xi, \eta)$. One can define a linear map $J$ on the tangent space of $M \times I R$ by

$$
J\left(X, f \frac{d}{d t}\right)=\left(\phi X-f \xi, \eta(X) \frac{d}{d t}\right)
$$

where $\mathrm{X}$ is a vector field in $\chi(M), t \in I R$ and $f$ is a real volume function. This indicates that $J^{2}=-I$. $J$, which is said to be an almost complex structure on $M \times I R$. $J$ is an integrable if its Nijenhuis torsion $N_{J}$ vanishes, where

$$
N_{J}=-[X, Y]+[J X, J Y]-J[J X, Y]-J[X, J Y] .
$$

$J$ is integrable if and only if $N_{J} \equiv 0$. It is well known that the almost contact structure $(\phi, \xi, \eta)$ is normal if and only if $N^{1} \equiv 0$, where

$$
N^{1}(X, Y)=N_{\phi}(X, Y)+2 d \eta(X, Y) \xi .
$$

A $(2 n+1)$-dimensional manifold $M$ is said to be a Sasakian manifold if it is endowed with a normal contact metric structure $(\phi, \xi, \eta, g, \varepsilon)$. It is well known that $M$ is a Sasakian structure if and only if $[4,5,7]$

$$
\left(\nabla_{X} \phi\right) Y=\varepsilon g(X, Y)-\eta(Y) X .
$$


Let us take a regular curve

$$
\gamma: I \subset I R \rightarrow E^{n}
$$

with $(I, \gamma)$ coordinate neighborhood, and its $\mathrm{i}-$ th curvature $k_{i}$ takes the following form as,

$$
\begin{aligned}
k_{i} & : I \rightarrow I R, \quad i=1,2, \ldots, n, \\
& s \rightarrow k_{i}(s)=g\left(V_{i}^{\prime}, V_{i+1}\right), \quad k_{n-1} \neq 0,
\end{aligned}
$$

where $\left\{V_{1}, V_{2}, \ldots, V_{n}\right\}$ is a Frenet $\mathrm{n}$-frame of the curve $\gamma$ [7].

\section{Normal and Osculating Curves In The 3-Dimensional Sasakian Space}

Theorem 3.1. Let $M$ be a 3-dimensional manifold endowed with the contact metric structure $(\phi, \xi, \eta, g, \varepsilon)$ then $M$ is a Sasakian manifold if and only if the torsion of its Legendre curves is $\varepsilon$. Let $\gamma$ be an arc-length parametrized curve on $M$, then the Frenet euations of $\gamma$ can be given partially as follows [3,4,7].

$$
\begin{aligned}
& \nabla_{V_{1}} V_{1}=\kappa V_{2}, \\
& \nabla_{V_{1}} V_{2}=-\kappa V_{1}+\varepsilon V_{3}, \\
& \nabla_{V_{1}} V_{3}=-V_{2} .
\end{aligned}
$$

Definition 3.1. Let $\gamma(s)$ be a regular curve in 3-dimensional Sasakian space. If the position vector of $\gamma(s)$ always lies in its normal plane, then it is called normal curve in 3-dimensional Sasakian space. Using this definition, one can write the following position vector of a curve $\gamma(s)$

$$
\gamma(s)=\xi(s) V_{2}(s)+\eta(s) V_{3}(s)
$$

where $\xi(s), \eta(s)$ are differentiable functions.

Theorem 3.2. Let $\gamma(s)$ be a regular curve in 3-dimensional Sasakian space, with curvature $\kappa(s), \varepsilon(s) \in R$. Then $\gamma(s)$ is a normal curve with $\kappa(s)=\frac{e^{\frac{\eta(s)}{\xi(s)}}}{\xi^{2}(s)}$ if and only if the principal normal curve and binormal components of the position vector are respectively given by

$$
\begin{aligned}
& <\gamma(s), V_{2}(s)>=\frac{-\kappa(s)}{\kappa^{2}(s)+\varepsilon(s)}+A_{1}(s) \cos (w s)+A_{2}(s) \sin (w s)+B_{1}(s) \cos (2 w s) \\
& +B_{2}(s) \sin (2 w s)+C_{1}(s) \cos (3 w s)+C_{2}(s) \sin (3 w s)
\end{aligned}
$$

and

$$
\begin{aligned}
& <\gamma(s), V_{3}(s)>=a_{1} \cos (w s)+a_{2} \sin (w s)+b_{1} \cos (2 w s) \\
& +b_{2} \sin (2 w s)+c_{1} \cos (3 w s)+c_{2} \sin (3 w s)
\end{aligned}
$$

where

$$
\begin{gathered}
A_{1}(s)=\frac{\left(\varepsilon(s)+w^{2}(s)\right)}{2 \varepsilon(s)} a_{1}, \quad A_{2}(s)=\frac{\left(\varepsilon(s)+w^{2}(s)\right)}{2 \varepsilon(s)} a_{2}, \quad B_{1}(s)=\frac{\left(\varepsilon(s)+4 w^{2}(s)\right)}{2 \varepsilon(s)} b_{1}, \\
B_{2}(s)=\frac{\left(\varepsilon(s)+4 w^{2}(s)\right)}{2 \varepsilon(s)} b_{2}, C_{1}(s)=\frac{\left(\varepsilon(s)+9 w^{2}(s)\right)}{2 \varepsilon(s)} c_{1}, C_{2}(s)=\frac{\left(\varepsilon(s)+9 w^{2}(s)\right)}{2 \varepsilon(s)} c_{2} \\
w= \pm\left(\frac{1}{2} \kappa^{2}(s)+2 \varepsilon(s) \pm \frac{1}{2}\left(\kappa^{4}(s)+12 \kappa^{2}(s) \varepsilon(s)+20 \varepsilon^{2}(s)\right)^{\frac{1}{2}}\right)^{\frac{1}{2}}
\end{gathered}
$$

and $a_{1}, a_{2}, b_{1}, b_{2}, c_{1}, c_{2} \in R$. 
Proof. Let us assume that $\gamma(s)$ is a normal curve in 3-dimensional Sasakian space, then, from Definition 3.1,

$$
\gamma(s)=\xi(s) V_{2}(s)+\eta(s) V_{3}(s)
$$

If one differentiates this with respect to s and using equation (3.1) the final product becomes,

$$
V_{1}(s)=-\kappa(s) \xi(s) V_{1}(s)+\left(\xi^{\prime}(s)-\eta(s)\right) V_{2}(s)+(\varepsilon(s) \xi(s)-\eta(s)) \eta^{\prime}(s) V_{3}(s)
$$

Again differentiating equation (3.5) with respect to $s$ and by using equation (3.1), gives,

$$
\begin{aligned}
\kappa(s) V_{2}(s) & =\left(-\kappa^{\prime}(s) \xi(s)-2 \kappa(s) \xi^{\prime}(s)+\eta(s) \kappa(s)\right) V_{1}(s) \\
& +\left(-\kappa^{2}(s) \xi(s)+\xi^{\prime \prime}(s)-2 \eta^{\prime}(s)-\varepsilon(s) \xi(s)\right) V_{2}(s) \\
& \left.+2 \varepsilon(s) \xi^{\prime}(s)-\varepsilon(s) \eta(s)+\varepsilon^{\prime}(s) \xi(s)+\eta^{\prime \prime}(s)\right) V_{3}(s) .
\end{aligned}
$$

Consideration of the hypotesis of theorem, the equation (3.21) becomes,

$$
\begin{aligned}
\xi^{\prime \prime}(s)-2 \eta^{\prime}(s)-\left(\kappa^{2}(s)+\varepsilon(s)\right) \xi(s) & =\kappa(s) \\
\eta^{\prime \prime}(s)+2 \varepsilon(s) \xi^{\prime \prime}(s)-\varepsilon(s) \eta(s)-\varepsilon^{\prime}(s) \xi(s) & =0 .
\end{aligned}
$$

If this system is solved, the result appears as,

$$
\begin{aligned}
\xi(s) & =\frac{-\kappa(s)}{\kappa^{2}(s)+\varepsilon(s)}+A_{1}(s) \cos (w s)+A_{2}(s) \sin (w s)+B_{1}(s) \cos (2 w s) \\
& +B_{2}(s) \sin (2 w s)+C_{1}(s) \cos (3 w s)+C_{2}(s) \sin (3 w s)
\end{aligned}
$$

and

$$
\begin{aligned}
\eta(s) & =a_{1} \cos (w s)+a_{2} \sin (w s)+b_{1} \cos (2 w s) \\
& +b_{2} \sin (2 w s)+c_{1} \cos (3 w s)+c_{2} \sin (3 w s)
\end{aligned}
$$

which completes the proof.

Changes of normal curve for different values of $\kappa$ and $\varepsilon$ are given in Figure 1.

Definition 3.2. Let $\gamma(s)$ be a regular curve in 3-dimensional Sasakian space. If the position vector of $\gamma(s)$ always lies in its osculating plane, then it is called osculating curve in 3-dimensional Sasakian space. By this definition, for a curve in 3-dimensional Sasakian space, the position vector of a curve $\gamma(s)$ satisfies

$$
\gamma(s)=a(s) V_{1}(s)+b(s) V_{2}(s)
$$

where $a(s), b(s)$ are differentiable functions.

Theorem 3.3. Let $\gamma(s)$ be a regular curve in 3-dimensional Sasakian space, with curvature $\kappa(s), \varepsilon(s) \in R$. Then $\gamma(s)$ is a osculating curve with $\varepsilon(s)=-\frac{e^{\frac{a(s) \kappa(s)}{b(s)}}}{b^{2}(s)}$ if and only if the tangential normal and principal normal components of the position vector are given respectively by the following expressions.

$$
<\gamma(s), V_{1}(s)>=c_{1} \exp \left[ \pm\left(\frac{1}{2} \varepsilon(s)-\kappa^{2}(s) \pm \frac{1}{2}\left(\varepsilon^{2}(s)-8 \kappa^{2}(s) \varepsilon(s)\right)^{\frac{1}{2}}\right)^{\frac{1}{2}} s\right]
$$

and 

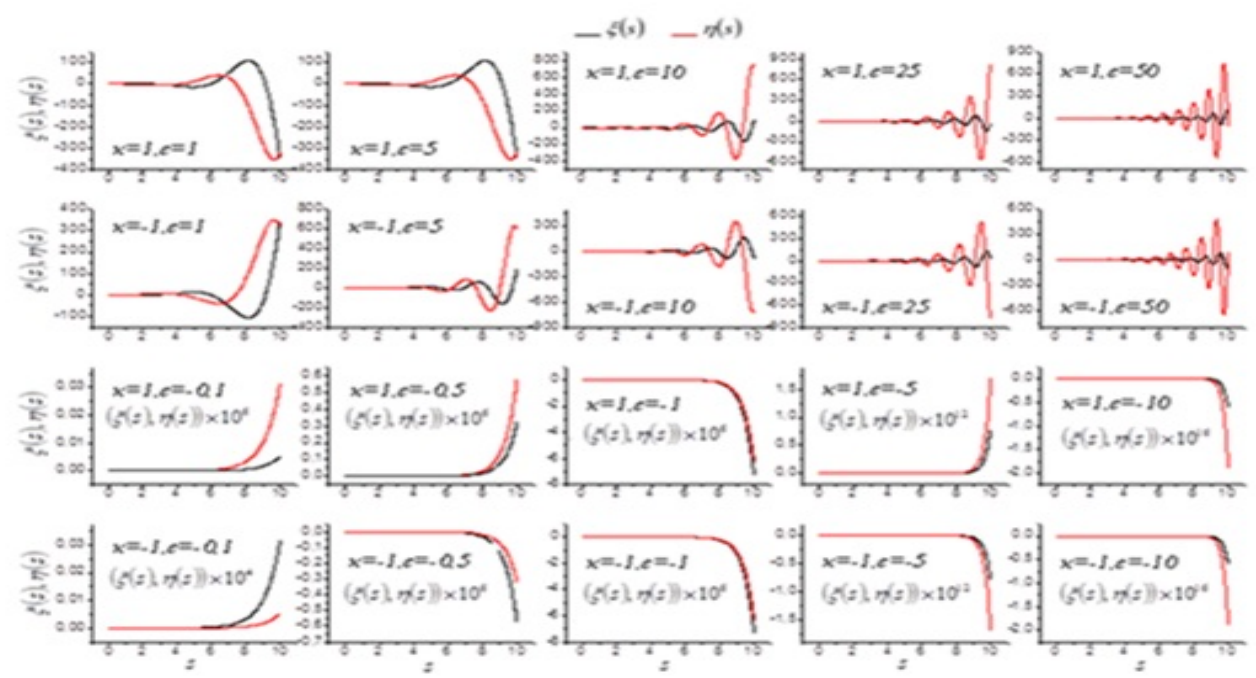

Figure 1. Normal curves which are given for different values of $\kappa(s)$ and $\varepsilon(s)$

$$
\begin{aligned}
& <\gamma(s), V_{2}(s)>=c_{1} \frac{\left[\left(\frac{1}{2} \varepsilon(s)-\kappa^{2}(s) \pm \frac{1}{2}\left(\varepsilon^{2}(s)-8 \kappa^{2}(s) \varepsilon(s)\right)^{\frac{1}{2}}\right)^{\frac{1}{2}}\right]}{2 \kappa(s)\left[ \pm\left(\frac{1}{2} \varepsilon(s)-\kappa^{2}(s) \pm \frac{1}{2}\left(\varepsilon^{2}(s)-8 \kappa^{2}(s) \varepsilon(s)\right)^{\frac{1}{2}}\right)^{\frac{1}{2}}\right]} \\
& \exp \left[ \pm\left(\frac{1}{2} \varepsilon(s)-\kappa^{2}(s) \pm \frac{1}{2}\left(\varepsilon^{2}(s)-8 \kappa^{2}(s) \varepsilon(s)\right)^{\frac{1}{2}}\right)^{\frac{1}{2}} s\right]+\frac{\kappa(s)}{\kappa^{2}(s)+\varepsilon(s)}
\end{aligned}
$$

where $c_{1} \in R$.

Proof. Let us assume that $\gamma(s)$ is a osculating curve in 3-dimensional Sasakian space, then, from Definition 3.2, one can write that (3.8).

Differentiating this with respect to s and using equation (3.1) leads to,

$$
V_{1}(s)=\left(a^{\prime}-\kappa(s) b(s)\right) V_{1}(s)+\left(a \kappa(s)+b^{\prime}(s)\right) V_{2}(s)+(\varepsilon(s) b(s)) V_{3}(s)
$$

Again differentiating this with respect to s and using equation (3.1) it is possible to obtain that,

$$
\begin{aligned}
\kappa(s) V_{2}(s) & =\left(a^{\prime \prime}(s)-\kappa^{\prime}(s) b(s)-\kappa(s) b^{\prime}(s)-a(s) \kappa^{2}(s)-\kappa(s) b^{\prime}(s)\right) V_{1}(s) \\
& +\left(a^{\prime}(s) \kappa(s)-b(s) \kappa^{2}(s)+a^{\prime}(s) \kappa(s)+a(s) \kappa^{\prime}(s)+b^{\prime \prime}(s)-\varepsilon(s) b(s)\right) V_{2}(s) \\
& +\left(a(s) \varepsilon(s) \kappa(s)+b^{\prime}(s) \varepsilon(s)+\varepsilon^{\prime}(s) b(s)+a(s) \kappa^{\prime}(s)+b^{\prime}(s) \varepsilon(s)\right) V_{3}(s) .
\end{aligned}
$$

Considering the hypotesis of theorem, equation (3.27) can be written as follows.

$$
\begin{aligned}
& a^{\prime \prime}(s)-\kappa^{\prime}(s) b(s)-\kappa(s) b^{\prime}(s)-a(s) \kappa^{2}(s)-\kappa(s) b^{\prime}(s)=0 \\
& b^{\prime \prime}(s)+\kappa^{\prime}(s) b(s)+2 \kappa(s) a^{\prime}(s)-b(s) \kappa^{2}(s)-\varepsilon(s) b(s)=\kappa(s) .
\end{aligned}
$$




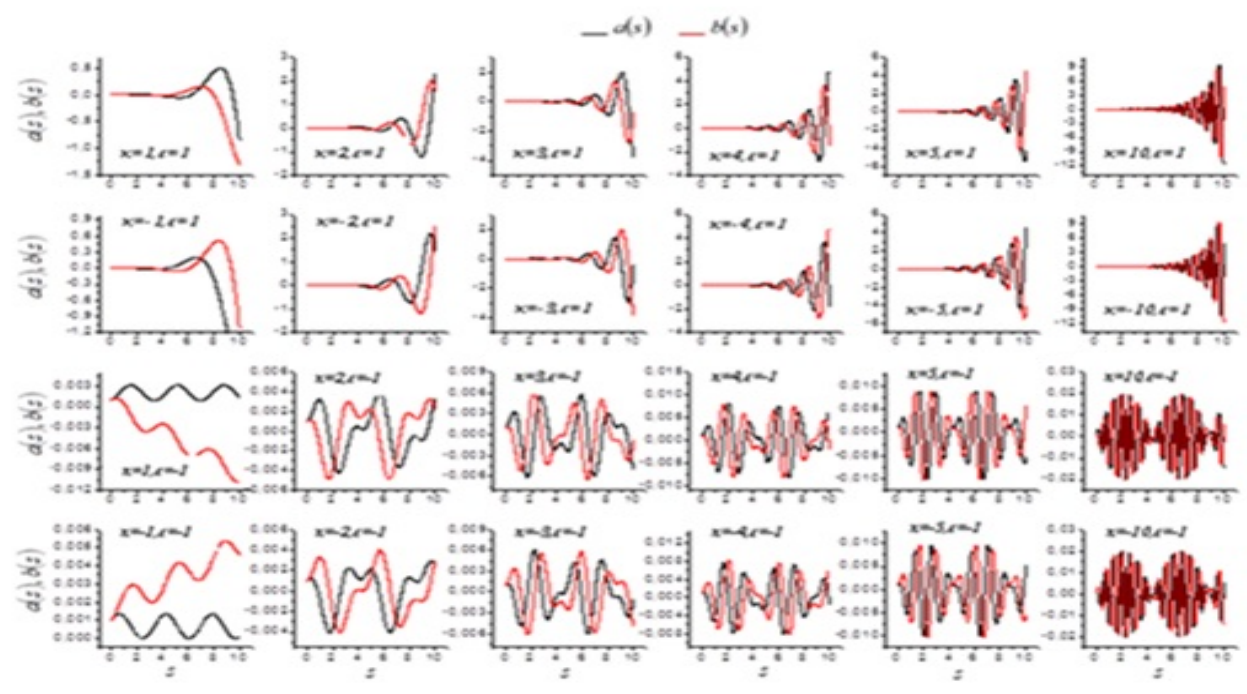

Figure 2. Osculating curves which are given for different values of $\kappa(s)$ and $\varepsilon(s)$

If this system is solved, the final product becomes,

$$
a(s)=c_{1} \exp \left[ \pm\left(\frac{1}{2} \varepsilon(s)-\kappa^{2}(s) \pm \frac{1}{2}\left(\varepsilon^{2}(s)-8 \kappa^{2}(s) \varepsilon(s)\right)^{\frac{1}{2}}\right)^{\frac{1}{2}} s\right]
$$

and

$$
\begin{gathered}
b(s)=c_{1} \frac{\left[\left(\frac{1}{2} \varepsilon(s)-\kappa^{2}(s) \pm \frac{1}{2}\left(\varepsilon^{2}(s)-8 \kappa^{2}(s) \varepsilon(s)\right)^{\frac{1}{2}}\right)^{\frac{1}{2}}\right]}{2 \kappa(s)\left[ \pm\left(\frac{1}{2} \varepsilon(s)-\kappa^{2}(s) \pm \frac{1}{2}\left(\varepsilon^{2}(s)-8 \kappa^{2}(s) \varepsilon(s)\right)^{\frac{1}{2}}\right)^{\frac{1}{2}}\right]} \\
\quad \exp \left[ \pm\left(\frac{1}{2} \varepsilon(s)-\kappa^{2}(s) \pm \frac{1}{2}\left(\varepsilon^{2}(s)-8 \kappa^{2}(s) \varepsilon(s)\right)^{\frac{1}{2}}\right)^{\frac{1}{2}} s\right]+\frac{\kappa(s)}{\kappa^{2}(s)+\varepsilon(s)}
\end{gathered}
$$

hence the theorem is proved.

Changes of osculating curve for different values of $\kappa$ and $\varepsilon$ are given in Figure 2. For $\varepsilon=-1, \kappa\rangle 1$ and $\varepsilon=-1$, $\kappa\langle-1$, it is observed that the waves are periodic. In addition frequencies are also nearly equal to each other.

\section{References}

[1] Alegre P., Blair D. E., Carriazo A, Generalized Sasakian Space Forms. Israel Journal of Mathematics 141 (2004), 157-183.

[2] Ali A.T., Onder M.A., Some Characterizations of Spacelike Rectifying Curves in the Minkowski Space-time. Global Journal of Science Frontier Research Mathematics \& Decision Sciences 12 (2012), no. 1.

[3] Baikoussis C., Blair D.E., On Legendre Curves In Contact 3-Manifolds. Geometria Dedicata 49 (1994), 135-142.

[4] Belkhelfa M., Hırıca I E., Rosca R., Verstraelen L., On Legendre curves in Riemannian and Lorentzian Sasaki spaces. Soochow J Math 28 (2002), no. 11, 81-91.

[5] Blair D.E., Contact Manifolds in Riemannian Geometry. Lecture Notes in Math. Berlin: Springer 509 (1976). 
[6] Blair D.E, Riemannian Geometry of Contact and Symplectic Manifolds. Boston: Birkhauser, 2002.

[7] Camcı Ç, Yaylı Y, Hacısalihoğlu H H, On the characterization of spherical curves in 3-dimensional Sasakian spaces. J Math. Anal. Appl.342 (2008), 1151-1159.

[8] Camcı Ç, Kula L., İlarslan K., Characterizations of the position vector of a surface curve in Euclidean 3-space. An Şt Ovidius Constanta 19 (2011), no. 3, 59-70.

[9] Cappelletti-Montano, B., Nicola, A., Yudin, I., Curvature Properties of Quasi-Sasakian Manifolds. International Journal of Geometric Methods in Modern Physics 10 (2013), no.8, 1360008.

[10] Chen B.Y., When does the position vector of a space curve always lie in its rectifying plane? Amer Math Monthly 110 (2003), 147-152.

[11] Chen B.Y., Dillen F., Rectifying curves as centrodes and extremal curves. Bull Inst Math Academia Sinica 33 (2005), no. 2, 77-90.

[12] Grbovic M., Nesovic E., Some relations between rectifying and normal curves in Minkowski 3-space. Math Commun. 17 (2012), 655-664.

[13] Güngör M.A., Tosun M., Some characterizations of quaternionic rectifying curves. Differential GeometryDynamical Systems 13 (2011), 89-100.

[14] İlarslan K., Spacelike normal curves in Minkowski space. Turk J. Math. 29 (2005), 53-63.

[15] İlarslan K., Nesovic E., Timelike and null normal curves in Minkowski space E 3 . Indian J. Pure Appl. Math. 35 (2004), 881-888.

[16] İlarslan K., Nesovic E., On Rectifying Curves as Centrodes and Extremal Curves in the Minkowski 3-space. Novi Sad J Math 37 (2007), no. 1, 53-64.

[17] İlarslan K., Nesovic E., Petrovic-Torgasev M., Some characterizations of rectifying curves in Minkowski 3-space. Novi Sad J Math.33 (2003), no :2, 23-32.

[18] Öztekin H., Öğrenmiş A.O., Normal and Rectifying Curves In Pseudo-Galilean Space G ${ }_{3}^{1}$ and Their Characterizations. J Math Comput. Sci. 2 (2012), no. 1, 91-100.

[19] Sasaki S., On differentiable manifolds with certain structures which are closely related to almost contact structure I. Tohoku Math J. 2 (1960), 459-476.

[20] Sharma, R., Ghosh, A., Sasakian 3-Manifold As a Ricci Soliton Represents in the Heisenberg Group. International Journal of Geometric Methods in Modern Physics 8 (2011), no. 1, 149-154.

[21] Yılmaz, M.Y., Külahc1, M., The Classifications of Quaternionic Osculating Curves In $Q^{4}$. Georgian Mathematical Journal (2017), 1-6.

\section{Affiliations}

MIHRIBAN ALYAMAÇ KÜLAHCI

ADDRESS: Department of Mathematics, Firat University, 23119 ELAZIĞ/TÜRKİYE.

E-MAIL: mihribankulahci@gmail.com

ORCID ID: 0000-0002-8621-5779

MEHMET BEKTAŞ

AdDRESS: Department of Mathematics, Firat University, 23119 ELAZIĞ/TÜRKİYE.

E-MAIL: mbektas@firat.edu.tr

ORCID ID: 0000-0002-5797-4944 
AHMET BİLİĊ

AdDRESS: Bartın University, Bartın/TÜRKIYYE.

E-MAIL: abilici@bartin.edu.tr

ORCID ID: 0000-0002-3192-456X 